\title{
Berufliches Selbstverständnis und Beanspruchung in der Schulleitung
}

\author{
Julia Warwas
}

Zusammenfassung: Der Beitrag setzt sich mit dem beruflichen Selbstverständnis schulischer Führungskräfte und ihren beruflichen Beanspruchungsprofilen auseinander, die mittels einer standardisierten Befragung bei $n=861$ Schulleiter(inne)n erhoben wurden. Zunächst wird die theoretische Basis der Untersuchung anhand eines Rahmenmodells schulischen Leitungshandelns skizziert. Erste empirische Befunde des Forschungsprojekts werden berichtet, die Vorstellungen einer homogenen Berufsgruppe widersprechen. Clusteranalytisch lassen sich fünf Gruppen von Leitungspersonen identifizieren, die nicht nur divergente Berufsauffassungen besitzen, sondern sich auch systematisch in ihrem Zufriedenheits- und Belastungserleben unterscheiden.

Schlüsselwörter: Berufliches Selbstverständnis · Berufliche Belastung ·

Berufliche Zufriedenheit $\cdot$ Rolle $\cdot$ Schulleitung

\section{Professional self-concept and the demands on school heads}

\begin{abstract}
This contribution investigates the professional self-concept of school heads and the demands on their professional competencies using a standardized survey of 861 school leaders. The paper starts with a sketch of the theoretical basis of the investigation, which is a framework model for school leadership. The initial findings of the research project contradict the concept of a homogeneous group of professionals. Using cluster analysis it is possible to identify five leadership groups, which not only display divergent occupational concepts, but differ systematically in their satisfaction and their experience of stress.
\end{abstract}

Keywords: Occupational stress · Occupational satisfaction $\cdot$ Professional self-concept $\cdot$ Role $\cdot$ School heads

Online publiziert: 21.08 .2009

(C) VS-Verlag 2009

Dipl.-Hdl. J. Warwas $(\bowtie)$

Lehrstuhl für Wirtschaftspädagogik, Otto-Friedrich-Universität Bamberg, Kärntenstraße 7, 96052 Bamberg, Deutschland

E-Mail: Julia.Warwas@uni-bamberg.de 


\section{Problemstellung}

Im Zuge einer erweiterten Selbstständigkeit der Einzelschule wird vermehrt die Forderung nach einem qualitativ neuartigen Rollenprofil schulischen Leitungspersonals laut, dessen definitorischen Minimalkonsens die Ablösung des reaktiv-verwaltenden Behördenvorstandes durch eine aktiv-gestaltende Führungspersönlichkeit bildet (vgl. z.B. Dubs 2005; Rosenbusch 2005; die Beiträge in Buchen \& Rolff 2006). Das empirisch gesicherte Wissen über das berufliche Selbstverständnis dieser Rollenträger ist jedoch verglichen mit der Fülle konzeptioneller Beiträge noch defizitär (vgl. z.B. Werle 2001, S. 98). Forschungsmethodisch bedarf es in diesem Zusammenhang nicht nur der Kontrolle personaler und kontextueller Einflüsse, die moderierend auf das schulleitende Handeln wirken, sondern auch der Auswahl und operationalen Erfassung geeigneter Erfolgsmerkmale der Leitungstätigkeit (vgl. z.B. Bonsen 2006).

Zufriedenheit im Beruf und Widerstandskraft gegenüber berufsspezifischen Belastungen gelten als wichtige Voraussetzungen qualitativ hochwertiger Arbeitsleistungen (vgl. z.B. Schaarschmidt \& Fischer 2001; Brägger \& Posse 2007) und erlauben einen ersten Zugriff auf relevante Zielgrößen des Leitungshandelns. Im Mittelpunkt dieses Beitrags steht deshalb die Frage, über welches berufliche Selbstverständnis schulische Leitungspersonen verfügen und ob divergente Ausprägungen dieses beruflichen Selbstverständnisses mit unterschiedlichen berufsbedingten Beanspruchungsprofilen einhergehen. In den empirischen Analysen werden dabei auch ausgewählte personen- und kontextspezifische Variablen als potenziell Bedingungsfaktoren berücksichtigt.

\section{Konzeptionelle Einbettung der Untersuchung}

\subsection{Rahmenmodell schulischen Leitungshandelns}

Die berichteten Auswertungen stellen erste Befunde eines umfangreichen Forschungsprojekts dar (vgl. ausführlich Warwas, in Vorbereitung), dem als analytischer Rahmen ein Modell zentraler Bestimmungsgrößen der Schulleitungstätigkeit zugrunde liegt. Das Modell wurde in Anlehnung an theoretische Entwürfe zum Handeln individueller Akteure im Bildungswesen (vgl. insbes. Fend 2006; Sembill 2006; Zlatkin-Troitschanskaia 2006) entwickelt und nimmt die Situationsspezifik der Leitungspraxis sowie deren subjektive Deutung und Gestaltung durch den Stelleninhaber explizit in den Blick. Ein wesentliches Konstruktionsprinzip des Modells besteht in der Kontrastierung von normierenden Einflussfaktoren auf der gesellschaftlichen Ebene mit divergenten individuellen Leistungsvoraussetzungen und variierenden organisationalen Handlungskontexten. Während Erstere in Form von kodifizierten Rechts- und Verwaltungsvorschriften, bildungspolitischen Steuerungsprogrammen und Qualifizierungsmodellen das Denken und Handeln schulischer Führungskräfte reglementieren und auf einheitliche Berufsstandards verpflichten, erzeugen Letztere ein Variationsspektrum an Realisationsformen der beruflichen Aufgabenerfüllung innerhalb des normativ strukturierten Handlungskorridors (vgl. Abb. 1). ${ }^{1}$ 


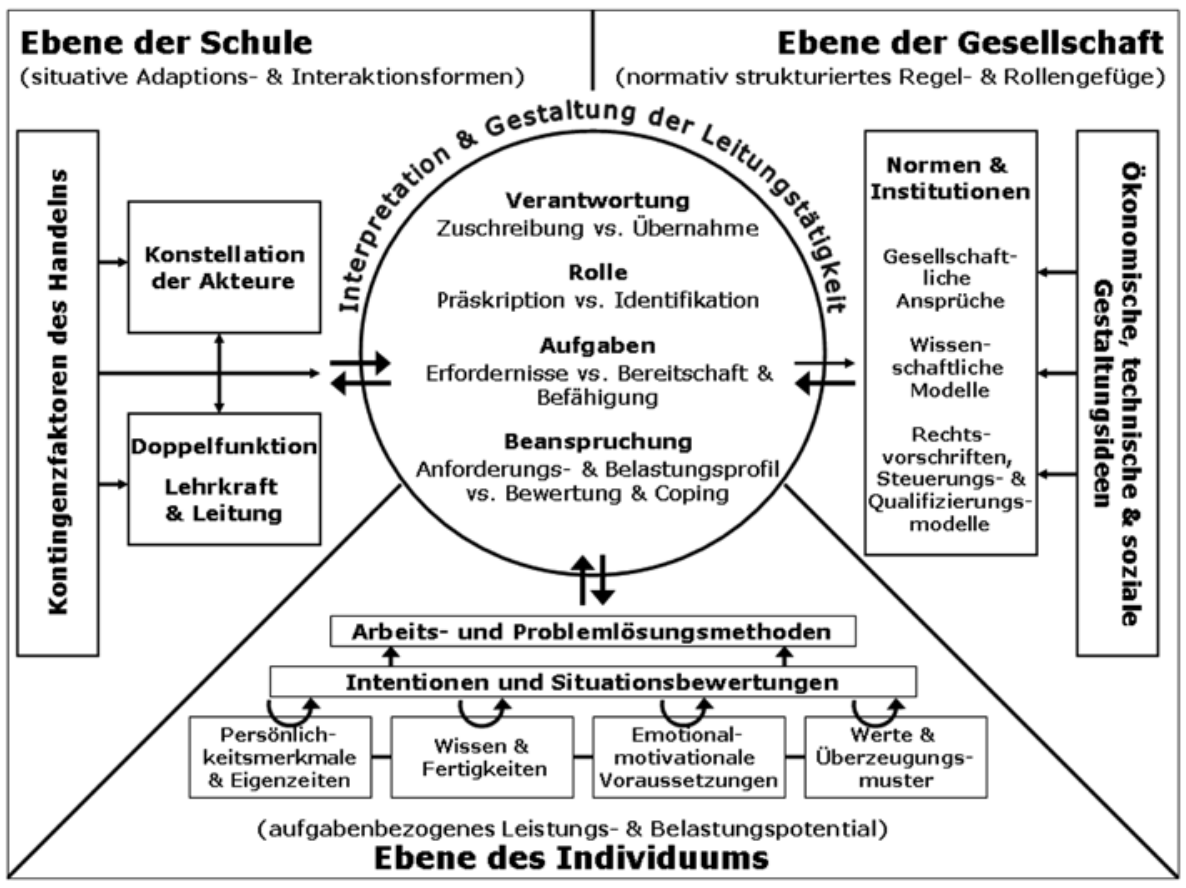

Abb. 1: Bestimmungsgrößen schulischen Leitungshandelns (in Anlehnung an Fend 2006; Sembill 2006)

Auf der Ebene der Einzelschule sieht sich jede Führungskraft distinkten Handlungserfordernissen, Gelegenheitsstrukturen und Problemlagen gegenüber. Diese resultieren beispielsweise aus der Größe sowie der materiellen und finanziellen Ausstattung der Organisation (Kontingenzfaktoren), aber auch aus den gegenseitigen Erwartungen, professionellen Handlungslogiken und arbeitsteiligen Strukturen, die sich im Laufe der Zeit in den Interaktionen der Schulmitglieder herauskristallisieren (Konstellation der Akteure). Die eigene Positionsbestimmung wird dabei durch die Doppelfunktion als Leitungs- und Lehrkraft erschwert, wobei die Relation der hiermit verbundenen Aufgabeninhalte ebenso wie die Akteurskonstellation von schulspezifischen Arbeitsbedingungen, z.B. einem nach Schülerzahlen gestaffelten Unterrichtsdeputat oder der Personalstruktur der Schule, beeinflusst werden.

Die Art und Weise, wie eine Führungskraft ihren beruflichen Auftrag interpretiert und handelnd ausgestaltet, lässt sich zudem mit ihrem individuellen, aufgabenbezogenen Leistungs- und Belastungspotenzial begründen, das sich z.B. aus berufsbezogenen Zielen, Kenntnissen und Fertigkeiten speist. Es ist davon auszugehen, dass sowohl die Berufsausübung als auch die berufsbedingte Beanspruchung in hohem Maße subjektiven Definitionen des Interaktionsraumes unterliegt, aus den verfügbaren persönlichen Ressourcen gespeist wird und vom aktualisierbaren Repertoire an Arbeits- und Problemlösestrategien der einzelnen Leitungsperson abhängt. Derartige interne Handlungsvoraussetzungen können in Anlehnung an die Kompetenzforschung (vgl. z.B. Baethge et al. 
2006) als komplexes Bündel angelegter und erworbener Dispositionen gefasst werden, die eine erfolgreiche Bewältigung beruflicher Anforderungssituationen ermöglichen.

\subsection{Definitorische Eingrenzung von beruflichem Selbstverständnis und berufsbedingter Beanspruchung}

Das berufliche Selbstverständnis (synonym: Berufsauffassung) wird angesichts des skizzierten Spannungsfeldes in Abgrenzung zum Konzept der beruflichen Rolle definiert. Letztere bündelt die Gesamtheit der formellen wie informellen, schematisierten Verhaltensanweisungen und normativen Verhaltensanforderungen, die von verschiedenen Seiten an den Inhaber einer bestimmten Position adressiert werden (vgl. z.B. Neuberger 2002, S. $314 \mathrm{ff}$.). Das berufliche Selbstverständnis umfasst dagegen die berufsbiografisch erworbenen Einstellungen und Verhaltensweisen des jeweiligen Positionsinhabers (vgl. z.B. Terhart 1996; Esslinger 2002), denen eine orientierende und handlungsleitende Funktion für die Alltagspraxis zuerkannt wird. Kognitive Anteile (z.B. Aufgabeninhalte), affektiv-evaluative Anteile (z.B. Erfolgsmaßstäbe) sowie dispositionelle Anteile (z.B. Habitus im Umgang mit Kollegen) bilden seine konstitutiven Bestandteile.

Mit Blick auf den inneren Kreis der Abb. 1 entsteht die individuelle Wahrnehmung und Ausgestaltung des Leitungsamtes folglich an den Schnittpunkten von persönlichen Entwürfen und fremddefinierten Ansprüchen (vgl. Krappmann 2005; Sembill 2006). Hier entscheidet sich, in welchem Umfang die einer Position zugeschriebene Verantwortung vom jeweiligen Inhaber auch übernommen wird, in welchen Bereichen sich die Person mit ihrem präskriptiven Tätigkeitsprofil identifiziert, dieses ablehnt oder zu verändern sucht, und inwieweit vorgegebene Aufgaben auf eine entsprechende Bereitschaft und (kompetenzbasierte) Befähigung des Individuums treffen. Da die Fremdbestimmung der Führungsrolle je nach Strukturierungsgrad der Situation stets gewisse Ermessens- und Gestaltungsspielräume zulässt, werden in der jüngeren Führungsforschung (vgl. zusammenfassend Steinmann \& Schreyögg 2005) Rollen nicht als starres Korsett betrachtet, das lediglich die konforme, passive Übernahme von Verhaltensnormen erlaubt (role taking). Vielmehr stellen sie Vereinbarungen dar, die zwischen dem Rollenträger und verschiedenen Kommunikationspartnern ausgehandelt werden (role making).

Diesen Ausschnitt des Rahmenmodells fokussiert auch der vorliegende Beitrag, in dem divergente Interpretations- und Gestaltungsvarianten beruflichen Rollenhandelns identifiziert und die hiermit verbundenen Beanspruchungsprofile ermittelt werden sollen. Negative Beanspruchungsreaktionen bzw. -folgen stellen sich gemäß transaktionaler Ansätze ein, wenn Arbeitsaufgaben und -bedingungen die adaptiven Mittel des Positionsinhabers übersteigen (vgl. z.B. Lazarus 1990; Rudow 2000). Diese Diskrepanz wird als subjektive Belastung wahrgenommen, die sich kurzfristig in Befindensbeeinträchtigungen wie Erschöpfung und langfristig in chronischen Krankheiten oder Burnout niederschlagen kann. Im Falle einer Passung von Anforderungsmerkmalen und Bewältigungsmöglichkeiten sind dagegen auch positive Beanspruchungsreaktionen und -folgen möglich (z.B. Wohlbefinden, das Gefühl von Leistungsfähigkeit und Gesundheit). 
Besonders in Positionen, die wie die Schulleitung zwischen vielfältigen Anspruchsgruppen lokalisiert sind (vgl. Dubs 2005; Seitz \& Capaul 2005), stellen die Pluralität, Widersprüchlichkeit, Uneindeutigkeit oder gar Unvereinbarkeit externer Verhaltenserwartungen potenzielle Belastungsfaktoren dar (vgl. auch Schmieta 2001, S. 85 ff.; Rudow 2000, S. 56; Neuberger 2002, S. 321 ff.). Um derartige Belastungen regulieren zu können, sind u.a. zwei Fähigkeiten nötig (vgl. Krappmann 2005, S. 40 ff.): Das Individuum muss nicht nur in der Lage sein, inkongruente Handlungsanforderungen als integralen Bestandteil interaktiver Arbeitsprozesse zu akzeptieren und auszuhalten (Ambiguitätstoleranz), sondern hierzu auch eine eigene Stellung beziehen, indem es den verschiedenen Rolleninhalten einen bestimmten Stellenwert in seinem subjektiven Berufsbild zuweist. Es benötigt demnach Rollendistanz als die Fähigkeit, sich über Rollenanforderungen ,zu erheben, um auszuwählen, negieren, modifizieren und interpretieren zu können“ (Krappmann 2005, S. 133). Dieser individuell zu leistende Balanceakt ist insbesondere dann zum Scheitern verurteilt, wenn das Individuum entweder bemüht ist, allen externen Erwartungen unter Missachtung bestehender Inkompatibilitäten und/oder unter Vernachlässigung der eigenen Bedürfnisstruktur gleichermaßen gerecht zu werden oder aber, wenn es versucht, einseitig eigene Bedürfnisse durch vollständige Zurückweisung der fremddefinierten Verhaltensanforderungen durchzusetzen.

Als möglicher Indikator einer gelungenen Balancierung wird hier die Berufszufriedenheit als individuelle Einstellung zur beruflichen Tätigkeit betrachtet, in der sich der Realisierungsgrad eigener Bedürfnisse, Wünsche und Ansprüche widerspiegelt (vgl. z.B. Ammann 2004). Dabei wird der theoretische Ansatz von Bruggemann, Groskurth und Ulich (1975) zugrunde gelegt, der eine interaktive Beziehung zwischen Person und Umwelt unterstellt. Demzufolge wägt ein Individuum eigene berufsbezogene Wünsche sowie Kennzeichen der gegebenen Arbeitssituation gegeneinander ab. Auf das Ergebnis dieser Soll-Ist-Wertbilanz kann es mit einer Erhöhung, Senkung oder Aufrechterhaltung seines Anspruchsniveaus reagieren. Eine vermittelnde Funktion nehmen individuelle Verarbeitungsstrategien ein, deren Wirksamkeit darüber entscheidet, ob es im Falle wahrgenommener Soll-Ist-Diskrepanzen zu einer Problemverdrängung, -fixierung oder -lösung kommt. Das Resultat der dynamischen Wechselwirkung zwischen Arbeitsplatz und anspruchsniveauregulierten Erwartungen sind qualitativ unterschiedliche Formen der Zufriedenheit (siehe hierzu Abschnitt 3.3), die in weitere Bewertungen einfließen.

\subsection{Fragestellungen, Prämissen und methodische Implikationen}

Erscheinungsformen und Determinanten der Beanspruchung schulischer Leitungspersonen wurden bis dato kaum systematisch analysiert (vgl. zum Forschungsstand z.B. Kranz 2007). Erste empirische Ergebnisse verweisen auf ein im Vergleich zur Berufsgruppe der Lehrkräfte eher moderates subjektives Belastungsniveau (vgl. z.B. Kanders \& Rösner 2006), legen jedoch eine Massierung von Stresssymptomen bei Grund- und Hauptschulleiter(inne)n nahe, die u.a. mangelnde externe Unterstützung und Anerkennung monieren, ein hohes Unterrichtsdeputat erfüllen müssen und aufgrund der unzureichenden personellen Auslastung des Sekretariats kaum Verwaltungsaufgaben delegieren können (vgl. Rosenbusch, Braun-Bau \& Warwas 2006). 
Stark unterbelichtet ist dagegen das Wirkungsgefüge zwischen berufsbezogenen Einstellungen, Wert- und Handlungsorientierungen und der beruflichen Beanspruchung, das bislang vorrangig bei Lehrkräften untersucht wurde (vgl. zusammenfassend z.B. Bieri 2006; Körner 2003). In dieser Berufsgruppe tragen laut einer vergleichenden Analyse verschiedener Studien „Rollenkonflikte und Rollenambiguitäten [...] definitiv zu Burnout bei“ (Schmieta 2001, S. 104; Auslassung: J.W.). Im Amt der Schulleitung evozieren insbesondere eine Aufgabenpluralisierung im Zuge bildungspolitischer Reformen, ein umfangreiches administratives Tätigkeitsspektrum sowie die Doppelfunktion als Leitungs- und Lehrkraft derartige konfligierende Handlungsanforderungen (vgl. z.B. Füssel 1998; Rolff 2006; Altrichter \& Rauscher 2008). Dadurch kollidieren z.B. die Aufgabe der Qualitätsentwicklung als innovationsfreudige Führungskraft mit der Verantwortung für die Einhaltung schulrechtlicher Bestimmungen als Behördenvorstand oder die Beurteilungs- und Kontrollfunktion eines (Dienst-)Vorgesetzten mit der Beratungsfunktion eines Coachs. Empirische Befunde nähren indes die Vermutung, dass dem „beachtlichen Frustrationspotential“" (Terhart 1997, S. 16) des Anforderungsprofils mit einer Flucht in berufsbiografisch erworbene Handlungsroutinen begegnet wird. Dementsprechend attestieren einige Untersuchungen schulischen Leitungspersonen eine Verhaftung im Ausgangsberuf der Lehrkraft (vgl. Storath 1995; Wissinger 1996; Werle 2001). Sie legen eine homogene Berufskultur nahe, in der die Schüler(innen) als primäre Bezugsgruppe gelten, erzieherische und verwaltende Tätigkeiten als zentrale Betätigungsfelder definiert werden und gegenüber dem Lehrerkollegium ein kollegial-egalitäres Verhältnis gepflegt wird (vgl. Bonsen 2006, S. 193; Kranz 2007, S. 92). Vereinzelt finden sich aber auch Hinweise auf schulart- und schulgrößenabhängige Varianzen. So manifestiert sich nach Baumert und Leschinsky (1986) im Selbstverständnis des primus inter pares, der administrative und repräsentative Zusatzfunktionen innehat, eine „spezifische Volksschultradition“ (a.a.O., S. 258). Nach Rolff (2006, S. 526) ist diese Handlungsorientierung vorrangig an kleinen Schulen zu finden.

Weitere Indizien für eine Ko-Existenz unterschiedlicher beruflicher Subkulturen liefern qualitative Interviewstudien, deren Ergebnisse aufgrund unterschiedlicher theoretischer Zugänge, Begriffsdefinitionen und Operationalisierungen allerdings nur beschränkt vergleichbar sind (vgl. z.B. Forberg 1997; Bonsen 2006; Languth 2006). Bei einer Untersuchung von 20 Schulleiter(inne)n identifiziert beispielsweise Languth fünf berufliche Orientierungsmuster, von denen aber nur eine der Handlungsrationalität einer Lehrkraft ähnelt. Die diesem Muster zurechenbaren Leitungspersonen sind in hohem Maße um sozio-emotionale Akzeptanz im Kollegium bemüht und empfinden die geringen Einflussmöglichkeiten ihrer Position in Kombination mit einer ausbleibenden Anerkennung ihrer Arbeit als stark belastend. Neben dieser ,, resignativen “ Berufsauffassung findet sich aber auch eine ,professionelle“ Berufsauffassung, für die eine hohe Identifikation mit der Leitungsfunktion, eine aktiv gestaltende Handlungsweise und die Fokussierung auf eine langfristige Sicherung guten Unterrichts kennzeichnend ist. Dagegen ermöglicht eine langjährige Berufserfahrung dem ,pragmatischen “ Schulleiter, gelassen mit Konflikten und Widersprüchen in der Alltagspraxis umzugehen, klare Prioritäten zu setzen und sich bei Wahl seiner Ziele und Mittel am Nützlichen und Machbaren zu orientieren.

Aus den skizzierten Modellannahmen und den empirischen Befunden ergeben sich für die folgenden Auswertungen vier Zielsetzungen: Erstens sollen Varianten des beruf- 
lichen Selbstverständnisses, die als divergente Muster der Interpretation und Gestaltung des beruflichen Rollenhandelns gefasst werden, identifiziert und beschrieben werden. Dabei erfolgt ein personenzentrierter, typologischer Zugriff unter Einsatz clusteranalytischer Verfahren, der ein differenzierteres Bild des angenommenen Variationsspektrums ergeben sollte als eine dimensionale Analyse einzelner Elemente der Berufsauffassung (vgl. auch Mayer, Filipp \& Aymanns 2004, S. 97). Entsprechend der Definition eines Typus, den eine spezifische Kombination von Merkmalsausprägungen kennzeichnet (vgl. Kluge 2000), interessiert bei der Analyse von Schulleitungstypen besonders die jeweils charakteristische Gesamtkonfiguration des individuellen Rollenhaushalts, die abgrenzbare Rolleninhalte subjektiv gewichtet und so in eine bestimmte Relation zueinander setzt.

Zweitens soll untersucht werden, ob ausgewählte personen- oder kontextgebundene Faktoren - namentlich Schulart, Schulgröße, Dienstalter und Geschlecht der Leitungskräfte - die ermittelten Muster von Berufsauffassungen beeinflussen. Besondere Aufmerksamkeit soll drittens der Frage zuteil werden, ob mit divergenten Berufsauffassungen auch Unterschiede in verschiedenen Facetten des Zufriedenheits- und Belastungserlebens einhergehen. Negative Auswirkungen auf die berufliche Beanspruchung werden insbesondere bei zwei problematischen Konstellationen erwartet, die ungelöste Rollenkonflikte bzw. fehlende Rollendistanz indizieren: Im Falle einer einseitigen Betonung der Wert- und Handlungsmuster einer Lehrkraft mit administrativen Zusatzfunktionen unter Zurückweisung der steuernden und koordinierenden Funktionen der Leitungsposition sind Enttäuschungen und resignative Tendenzen wahrscheinlich, da eigene Erwartungen und Bedürfnisse mit dem normativen Anforderungsprofil des Berufs nur bedingt in Einklang gebracht werden können. Dieser Typus dürfte in Volksschulen sowie in kleinen Schulen dominieren. Im Unterschied hierzu dürfte ein Bemühen um größtmögliche Konformität mit allen Rollensegmenten bzw. mit den Erwartungen sämtlicher Interaktionspartner im beruflichen Alltag nicht realisierbar sein, ohne ,,in einer Welt ohne Normenkonsens zerrissen zu werden“ (Krappmann 2005, S. 80). Vielmehr dürfte diese Strategie aufgrund inkompatibler und widersprüchlicher Handlungsanforderungen in eine Selbstüberforderung münden. $\mathrm{Zu}$ erwarten ist sie bei Berufsanfängern, die sich einseitig an normativen Berufsleitbildern orientieren und idealisierende Machbarkeitsüberzeugungen hegen, ohne sich struktureller Restriktionen oder situativer Erfordernisse bewusst zu sein (vgl. hierzu Roggenbuck-Jagau 2005, S. 297). Sie dürfte weiterhin gehäuft an kleinen Schulen auftreten, an denen die Leitungskraft aufgrund fehlender Funktions- und Verwaltungsstellen oft als „Mädchen für alles“ fungieren muss.

Sowohl das skizzierte Rahmenmodell als auch die referierten Forschungsergebnisse verweisen darauf, dass berufliches Selbstverständnis und berufsbedingte Beanspruchung durch die Wirkung bestimmter Bedingungsfaktoren konfundiert sein können. Deshalb soll in einem letzten Analyseschritt durch Kovarianzanalysen der Einfluss der Schulart und -größe sowie des Dienstalters und des Geschlechts bei der Erklärung von Unterschieden im allgemeinen Zufriedenheits- und Belastungserleben kontrolliert werden. Dieses strenge Verfahren soll aufdecken, ob spezifische Muster von Berufsauffassungen einen zusätzlichen, von allgemeinen Merkmalen der Person und des Handlungskontexts unabhängigen Erklärungsbeitrag leisten können. 


\section{Methode}

\subsection{Datenerhebung und Stichprobe}

An der standardisierten schriftlichen Befragung auf dem Postweg nahmen bayerische Schulleiter(innen) verschiedener Schularten teil. Um ausreichend große Zellenbesetzungen für Schulartvergleiche zu gewährleisten, wurde eine Quotenstichprobe gezogen. Nach der Eliminierung von 10 Fragebögen mit systematischen Missings stützen sich die Auswertungen auf die Antworten von 861 Führungskräften (Rücklaufquote 57\%), von denen 789 das Amt des Schulleiters und 53 das des Stellvertreters bekleiden (19 Personen machten keine Angaben). Die Befragten sind im Mittel 56 Jahre alt $(\mathrm{SD}=5,61)$ und durchschnittlich seit 8 Jahren $(\mathrm{SD}=6,22)$ in diesen Positionen. In der Stichprobe befinden sich überwiegend männliche Teilnehmer (80\%), was der Unterrepräsentanz von Frauen im Leitungsberuf geschuldet ist (vgl. hierzu Hobeck 2005). Rund 36\% der Befragten sind an Grund- und Hauptschulen tätig, etwa 23\% an Gymnasien. 20\% der Probanden leiten Realschulen und weitere 20\% stehen berufsbildenden Schulen vor. Die Schulen werden im Schnitt von 749 Schüler(inne)n besucht, wobei die Stichprobe alle OrganisationsgröBen umfasst $(\mathrm{SD}=582,60 ; \mathrm{Min}=45 ; \operatorname{Max}=4000)$.

\subsection{Erfassung der beruflichen Rollen des schulischen Leitungspersonals}

Zur Operationalisierung verschiedener beruflicher Handlungsanforderungen der Schulleitung wurden aus Literaturanalysen und leitfadengestützten Interviews mit Schulleiter(inne)n Itembatterien entwickelt und auf der Basis einer Pilotstudie bei $n=40$ Schulen in Hessen überarbeitet. Die Items umfassen Einschätzungen zu Funktion, Aufgaben und Verantwortung der Schulleitung, ihrer Stellung gegenüber Kollegialorganen und ihrem Gestaltungspotenzial, die auf einem sechsstufigen Antwortformat (,trifft gar nicht zu“ bis „trifft sehr zu“) abgegeben werden. Über die zwölf in der Hauptuntersuchung eingesetzten Items wurde eine orthogonale Faktorenanalyse (Varimax-Rotation) gerechnet. Unter Zugrundelegung des Kaiser-Kriteriums (Eigenwerte $>1$ ) wurden dabei drei Faktoren extrahiert, die zusammen 50\% der Varianz erklären und als idealtypische Rollenmuster interpretierbar sind. Die Bezeichnungen, Kennwerte und Beispielitems der gebildeten Skalen sind Tab. 1 zu entnehmen.

Die Skala Leadership korrespondiert mit einigen wesentlichen der im Konzept des Transformational Leadership definierten Handlungsanforderungen (vgl. Dubs 2005; Seitz \& Capaul 2005, S. 275 ff.). Die Leitungsperson fungiert als visionäre und mitreiBende Führungspersönlichkeit, deren Aktivitäten auf die Zielmarke verbesserter Schülerleistungen gerichtet sind. $\mathrm{Zu}$ ihren Hauptaufgaben gehören die Entwicklung und Kommunikation langfristiger gesamtschulischer Ziele, die Motivierung der Lehrkräfte zu außerordentlichem Engagement sowie die individuelle Beratung der Lehrkräfte auf Basis einer ausgeprägten pädagogischen Expertise. Dagegen beschreibt die Skala Kollegialität eine dem Autonomie-Paritätsmuster verpflichtete und auf dem Prinzip der Nicht-Einmischung basierende Handlungsorientierung, die üblicherweise Lehrkräften attestiert wird (vgl. Altrichter \& Eder 2004; Terhart 1996). Gleichberechtigung, Konsens und die strikte Beachtung der pädagogischen Freiheit aller Kollegen bilden die Leitwerte eines Han- 


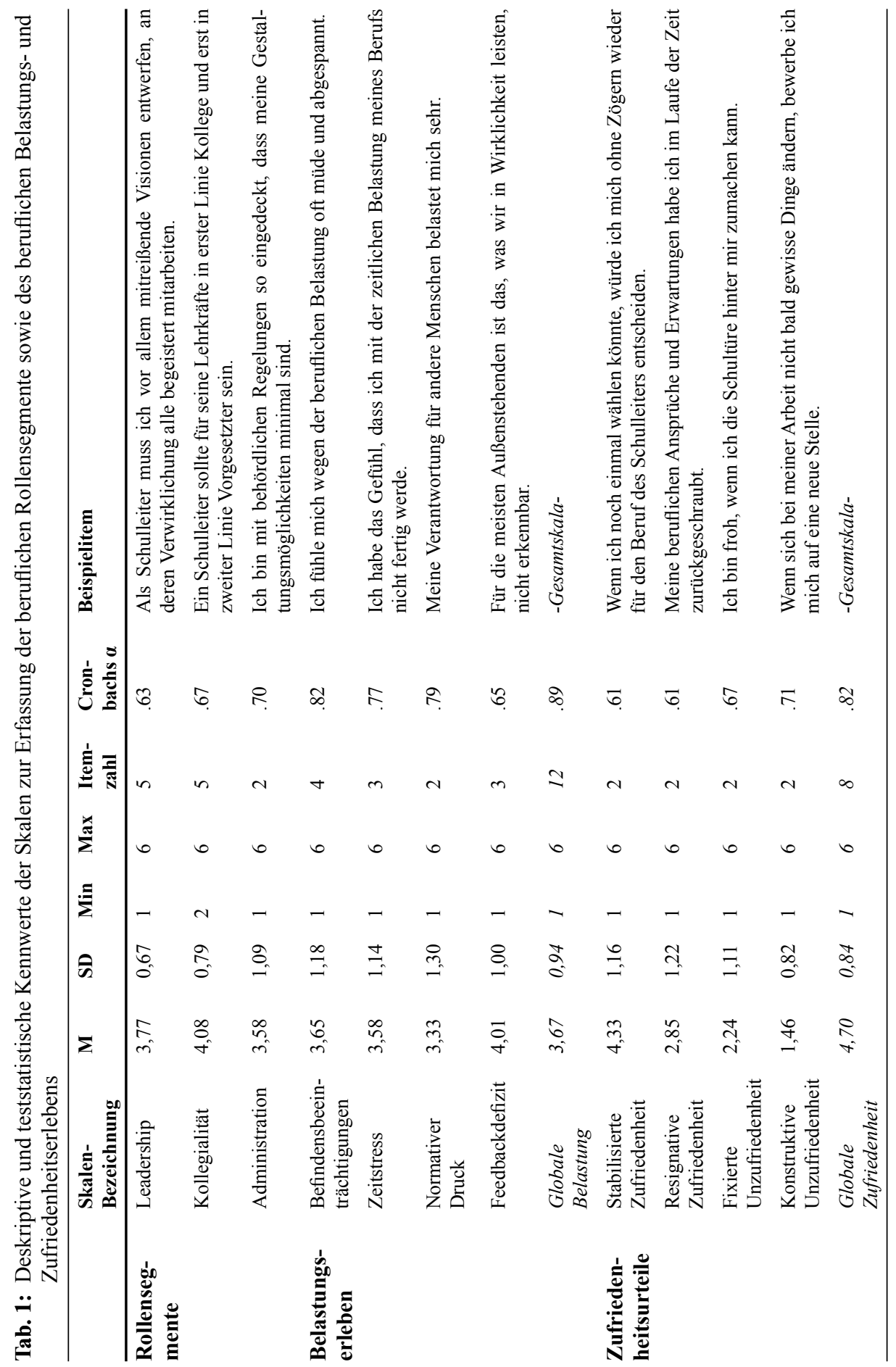


delns, das inhaltlich von den Kategorien der Unterrichts- und Erziehungstätigkeit dominiert wird. Im Umgang mit dem pädagogischen Personal ist die Leitungsperson um ein kollegiales Auftreten auf Augenhöhe bemüht, das formale Positionsunterschiede nivelliert und sich unauffällig im Hintergrund vollzieht. Die Skala Administration charakterisiert schließlich das Rollensegment des Behördenvorstandes mit minimalem Handlungsspielraum, dessen Funktion sich auf den reaktiven Vollzug behördlicher Vorgaben beschränkt. Tabelle 2 ist zu entnehmen, dass die gebildeten Skalen analytisch gut zu trennen sind.

\subsection{Erfassung der beruflichen Belastung und Zufriedenheit}

Die subjektive Belastung wurde mittels Fragebogenadaptionen von Dann et al. (2007) sowie Schwarzer und Jerusalem (2001) erhoben. Mithilfe der zwölf Items der Gesamtskala kann ermittelt werden, auf welchem Niveau sich das Belastungserleben schulischer Führungskräfte generell bewegt. Zudem geben vier Subskalen Aufschluss darüber, ob die Befragten entlang theoretisch unterscheidbarer Dimensionen spezifische Belastungsschwerpunkte aufweisen (vgl. Tab. 1). Dabei integriert die Kategorie Befindensbeeinträchtigungen verschiedene Symptome, die als Frühwarnsignale chronischer Krankheitsverläufe interpretiert werden können und ein eingeschränktes Wohlbefinden sowie eine wahrgenommene reduzierte Leistungsfähigkeit indizieren. Dagegen ist Zeitstress vom Eindruck geprägt, bei der Arbeit permanent zu Hast und Eile getrieben zu sein und zu wenig zeitliche Freiräume und Erholungspausen zu besitzen. Normativer Druck beinhaltet das Empfinden, uneinlösbaren fremddefinierten Erwartungen ausgesetzt und der Verantwortung des Amtes nicht gewachsen zu sein. Schließlich reflektiert Feedbackdefizit einen subjektiven Mangel an konkreten Erfolgen, das Ausbleiben von Anerkennung und positiven Rückmeldungen durch wichtige Bezugsgruppen sowie das Fehlen allgemein akzeptierter Bewertungskriterien der Berufsausübung.

Zur Operationalisierung der beruflichen Zufriedenheit wurde ebenfalls auf ein in Untersuchungen von Lehrkräften bewährtes Instrument zurückgegriffen (vgl. hierzu Bieri 2006, S. 116ff.), das für den Anwendungsbereich der Schulleitung modifiziert wurde. Über die Erfassung einer bilanzierenden Globaleinschätzung der beruflichen Situation hinaus erlauben auch hier vier Subskalen eine differenzierte Betrachtung theoretisch unterscheidbarer Zufriedenheitsformen (vgl. Tab. 1). Während die stabilisierte Zufriedenheit eine Erfüllung berufsbezogener Bedürfnisse und den Wunsch nach Beibehaltung des erreichten Zustandes impliziert, resultiert die resignative Zufriedenheit nicht aus einer gelungenen Bedürfnisbefriedigung, sondern vielmehr aus einer Minderung des Anspruchsniveaus, auf dessen Basis vorgefundene Arbeitsbedingungen beurteilt werden. Fixierte Unzufriedenheit stellt sich ein, wenn eine als unbefriedigend erlebte Arbeitssituation passiv als ausweglos hingenommen wird, wohingegen sich die konstruktive Unzufriedenheit durch aktive Problemlösungsversuche auszeichnet, um die gegebenen Arbeitsbedingungen zu überwinden oder zu verlassen.

Tabelle 2 zeigt, dass die verschiedenen Zufriedenheitsqualitäten in der anzunehmenden Richtung korrelieren. Sie dokumentiert auch die große gemeinsame Varianz der Subskalen der subjektiven Belastung. Dabei stehen die Intensitäten des Zufriedenheits- und Belastungserlebens in einem reziproken Verhältnis zueinander. 


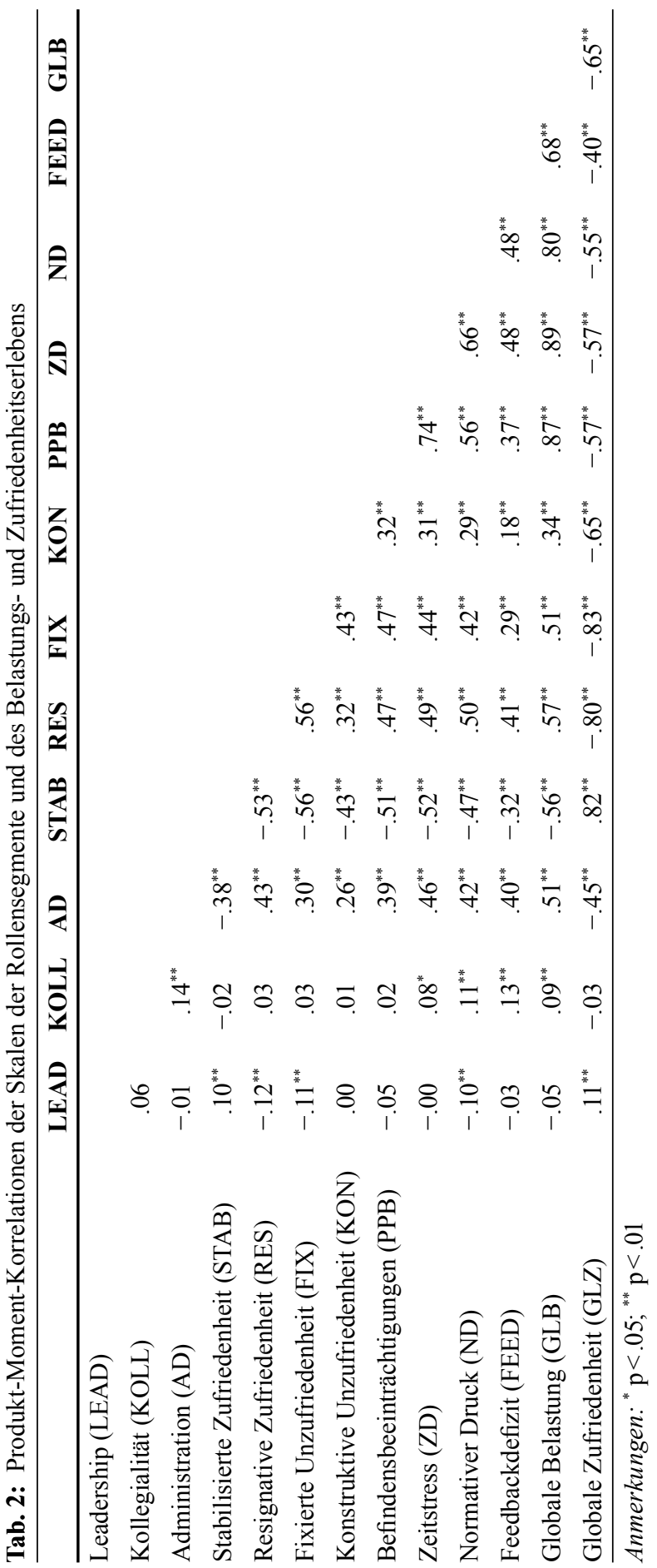




\section{Empirische Befunde}

\subsection{Berufsauffassungen von Schulleiter(inne)n}

Unter Einsatz clusteranalytischer Verfahren konnten fünf homogene Gruppen schulischer Leitungskräfte identifiziert werden, die sich in der subjektiven Gewichtung beruflicher Rollensegmente systematisch unterscheiden (vgl. Abb. 2). Hierzu wurden zunächst $n=300$ Personen einer hierarchisch-agglomerativen Clusteranalyse nach dem Ward-Algorithmus über die drei standardisierten Faktorwerte der berichteten Faktorenanalyse unterzogen. Da der Verlauf der Fehlerquadratsummen beim Übergang von der 5- zur 4-Clusterlösung den stärksten Heterogenitätszuwachs verzeichnete, wurden fünf Gruppen gebildet. Um deren interne Homogenität zu maximieren, schloss sich in der Gesamtstichprobe eine iterativ-partitionierende Clusterzentrenanalyse nach dem k-means Algorithmus an. Als Indizien der Güte dieser optimierten Lösung können gewertet werden (vgl. Bacher 1996; Bortz 2005, S. 580 ff.):

- die teststatistische Absicherung der 5-Clusterlösung: Ein Vergleich verschiedener, mittels k-means Verfahren erzeugter Clusterlösungen im Hinblick auf die jeweils erklärte Streuung $\left(\mathrm{ETA}_{\mathrm{k}}^{2}\right)$, die relative Verbesserung gegenüber der vorausgehenden Lösung $\left(\mathrm{PRE}_{\mathrm{k}}\right)$ sowie anhand ihrer F-MAX-Statistik lässt sowohl eine 4- als auch eine 5-Clusterlösung als formal zulässig erscheinen;

- die inhaltlich sinnvolle Interpretierbarkeit der generierten Leitungstypen, deren unterschiedliche Rollenkonfiguration nachfolgend beschrieben werden. Sie bildete das endgültige Entscheidungskriterium für die 5-Clusterlösung;

- stabile Clusterzuordnungen unabhängig von der Startwertkonfiguration der Clusterzentren: Ein Vergleich der ursprünglichen Clusterlösung auf Basis der vom Statistikprogramm automatisch erzeugten Startwerte mit einer Lösung, in die eine zufällig generierte Ausgangspartition eingelesen wurde, weist eine gute Übereinstimmung von $\kappa=.718$ auf;

- gegenüber Stichprobeneffekten, robuste Gruppierungen der Objekte in zwei Teilstichproben: Eine Doppelkreuzvalidierung separat durchgeführter Clusteranalysen in zwei gleich großen Teilstichproben, in welche die Gesamtstichprobe zufällig unterteilt wurde, erzielt ebenfalls gute Übereinstimmungswerte von $\kappa=.808$ (zwischen A und $A^{*}$ ) sowie $\kappa=.792$ (zwischen B und $\mathrm{B}^{*}$ ) und spricht für die Generalisierbarkeit der Clusterlösung;

- ähnliche Muster von Berufsauffassungen in korrespondierenden Clustern der Teilstichproben: Da sich im Falle einer stabilen Lösung die Ausprägungen der Klassifikationsmerkmale korrespondierender Leitungstypen nicht unterscheiden sollten, wurden die Cluster beider Teilstichproben einer multivariaten Varianzanalyse über die drei Rollensegmente mit anschließendem Scheffé-Test unterzogen. Mit Ausnahme eines einzigen der fünf Vergleichspaare, das im Bereich Leadership keine homogene Untergruppe bildet, lassen sich dabei keine signifikanten Abweichungen feststellen;

- eine korrekte diskriminanzanalytische Klassifikation der Untersuchungsobjekte: Die Clusterzugehörigkeit der Elemente einer Teilstichprobe konnte anhand der in der jeweils anderen Teilstichprobe geschätzten Diskriminanzfunktion gut vorhergesagt werden. Dabei wurden im ersten Validierungsschritt 95,3\%, im zweiten 95,2\% der nicht für die Funktionsberechnung herangezogenen Objekte richtig zugeordnet. 


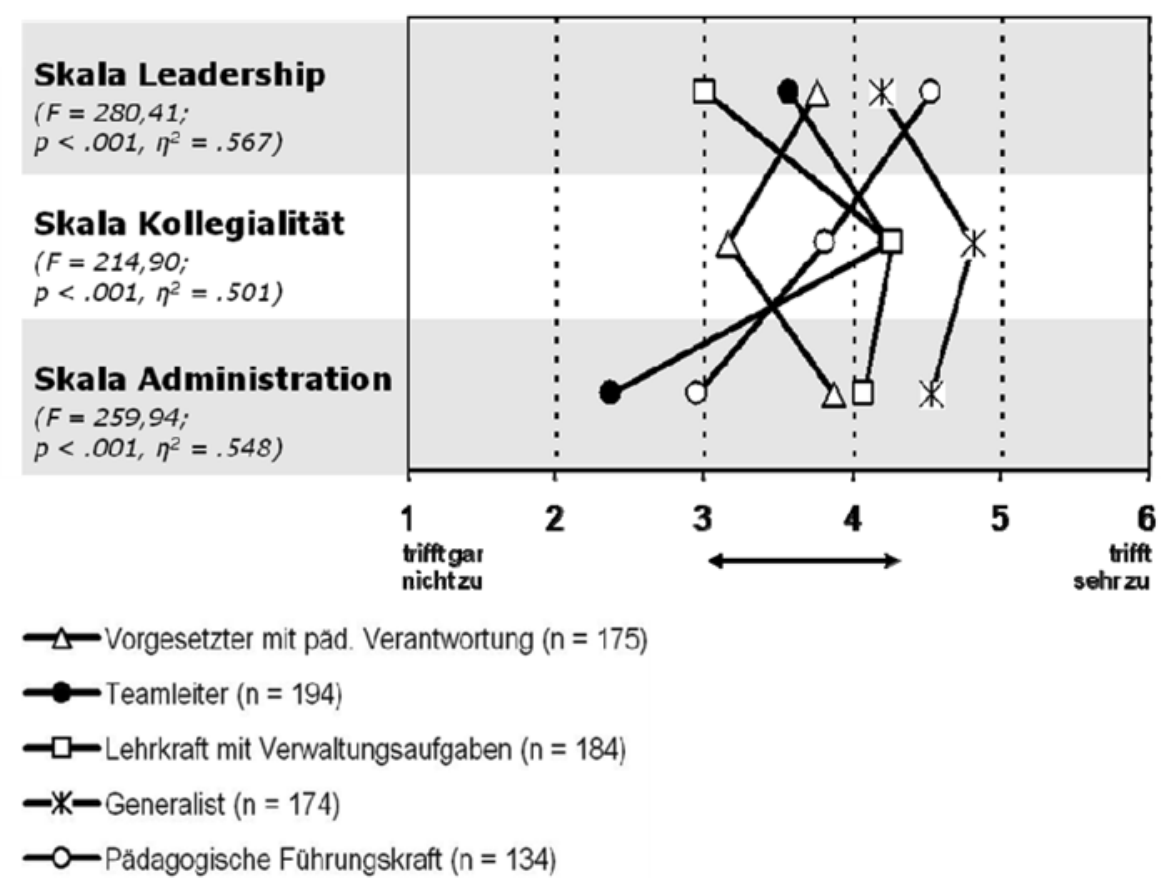

Abb. 2: Varianten des subjektiven Berufsverständnisses von Schulleiter(inne)n (5-Cluster-Lösung)

Die mit $n=134$ kleinste Gruppierung weist eine hohe Identifikation mit den für Führungspositionen konstitutiven Funktionen der Zielbildung, Steuerung, Motivation und Beratung auf, die in der Skala Leadership gebündelt sind $(\mathrm{M}=4,54 ; \mathrm{SD}=0,43)$. Eine kollegiale Handlungsorientierung nimmt demgegenüber einen deutlich geringeren und in der Gesamtstichprobe unterdurchschnittlichen Stellenwert ein $(\mathrm{M}=3,82 ; \mathrm{SD}=0,58)$. Klar nachrangig wird die Bedeutung reaktiv-vollziehender Verwaltungsaktivitäten eingestuft $(M=2,96 ; S D=0,75)$. Da dieser Leitungstyp auf einen klaren Führungsanspruch mit inhaltlicher Richtlinienkompetenz unter deutlicher Zurückweisung administrativer Aufgaben schließen lässt, wird er als „Pädagogische Führungskraft“ bezeichnet.

Im Gegensatz hierzu räumt die „Lehrkraft mit Verwaltungsaufgaben“ ( $n=184)$ Elementen der pädagogischen Führung das im Clustervergleich geringste Gewicht ein $(\mathrm{M}=3,02 ; \mathrm{SD}=0,47)$. Personen dieser Gruppierung scheinen eine derart herausgehobene, lenkende Position und den hiermit verbundenen Expertenstatus weitgehend abzulehnen und sehen sich stattdessen primär mit der Abwicklung bürokratischer Prozeduren befasst, die ihre gestalterische Einflussnahme auf die Schulqualität blockieren $(M=4,09$; $\mathrm{SD}=0,77)$. Einen hohen Stellenwert besitzen in ihrem Berufsverständnis zudem die harmonische Integration in das Kollegium, die Ausübung originär pädagogischer Funktionen und die Wahrung der Nicht-Einmischungsnorm in die Arbeit der anderen, gleichberechtigten Lehrkräfte, wie sie in der Skala Kollegialität zum Ausdruck kommen $(\mathrm{M}=4,28$; $\mathrm{SD}=0,58)$. 
Verglichen mit allen anderen Gruppen nimmt der ,, Vorgesetzte mit pädagogischer Verantwortung “ $(n=175)$ mit dem niedrigsten Wert auf der Skala Kollegialität das stärkste hierarchische Gefälle wahr und hat sich am weitesten von der Handlungsorientierung seines Ausgangsberufs entfernt $(\mathrm{M}=3,16 ; \mathrm{SD}=0,46)$. Aktiv-gestaltende Rolleninhalte $(\mathrm{M}=3,77 ; \mathrm{SD}=0,44)$ und vollziehend-administrative Elemente $(\mathrm{M}=3,88 ; \mathrm{SD}=0,82)$ halten sich in dieser Berufsauffassung die Waage. ${ }^{2}$

Einschränkungen der eigenen Gestaltungsspielräume durch restriktive behördliche Vorgaben werden von dem größten Cluster $(n=194)$ praktisch nicht wahrgenommen. Noch deutlicher als die Pädagogische Führungskraft weist diese Gruppierung die ausführende Verwaltungsfunktion der Schulleitung zurück $(\mathrm{M}=2,38 ; \mathrm{SD}=0,62)$. Zentralen Stellenwert besitzen in ihrem beruflichen Selbstverständnis die Handlungsprinzipien des Konsenses, der Gleichberechtigung und Eigenverantwortung der Kollegiumsmitglieder $(\mathrm{M}=4,26 ; \mathrm{SD}=0,67)$. Im Gegensatz zur Lehrkraft mit Verwaltungsaufgaben, mit der sie diese kollegiale Grundorientierung teilt, ${ }^{3}$ besitzt sie aber ein höheres Bewusstsein für Aufgaben der Zielbildung und Personalführung $(\mathrm{M}=3,57$; $\mathrm{SD}=0,45)$. Diese Konstellation legt die Vermutung nahe, dass sie eine pädagogische Qualitätsentwicklung nicht wie die pädagogische Führungskraft mit der Steuerungsintensität eines herausragenden Experten und strategischen Visionärs, sondern als partnerschaftlicher „Teamleiter “ betreiben möchte.

Die Berufsauffassung des „,Generalisten“ $(n=174)$ vereint schließlich inkonsistente Handlungsorientierungen und widerstreitende Handlungsanforderungen auf durchgängig weit überdurchschnittlichem Niveau. Die in diesem Cluster gebündelten Schulleiter(innen) fühlen sich mit Abstand am stärksten den als Autonomie-Paritätsmuster bekannten beruflichen Leitwerten einer regulären Lehrkraft verpflichtet $(M=4,82 ; \mathrm{SD}=0,46)$, beanspruchen jedoch gleichzeitig eine strategische Führungsposition im Kollegium $(M=4,19$; $\mathrm{SD}=0,42$ ) und stufen den reaktiven Vollzug gesetzlich-administrativer Regelungen als zentrales Betätigungsfeld ein $(\mathrm{M}=4,54 ; \mathrm{SD}=0,69)$.

\subsection{Einfluss ausgewählter personen- und kontextspezifischer Merkmale auf das} berufliche Selbstverständnis

Um zu überprüfen, ob die ermittelten Muster von Berufsauffassungen geschlechtsspezifisch, erfahrungsabhängig oder kontextgebunden auftreten, wurden $\chi^{2}$-Tests und Varianzanalysen durchgeführt. Diese weisen das Geschlecht der Leitungsperson als unbedeutend für die Clusterzugehörigkeit aus $\left(\chi^{2}=5,944 ; p=0.20\right)$, wobei zu berücksichtigen ist, dass die Stichprobe zum größten Teil aus männlichen Leitungspersonen besteht (vgl. Abschnitt 3.1). Auch Zusammenhänge zwischen dem beruflichen Selbstverständnis und der Berufserfahrung (gemessen an den Amtsjahren) lassen sich - entgegen der formulierten Annahme - nicht nachweisen $\left(\mathrm{F}=2,326 ; \mathrm{p}=.055, \eta^{2}=.011\right)$.

Signifikant und erwartungskonform fallen jedoch die Gruppenvergleiche im Hinblick auf die Schulart $\left(\chi^{2}=51,528 ; p<.001\right)$ und die Schulgröße $\left(F=5,459 ; p<.001, \eta^{2}=.025\right)$ aus. Dabei zeigt sich, dass jeweils knapp die Hälfte der in den Clustern Lehrkraft mit Verwaltungsaufgaben sowie Generalist gebündelten Personen an Volksschulen (d.h. Grund- und Hauptschulen) arbeiten. Demgegenüber fällt die Verteilung der Cluster Pädagogische Führungskraft sowie Teamleiter deutlich homogener aus: Sie sind mit Anteilen 
zwischen $22 \%$ und $28 \%$ bzw. zwischen $19 \%$ und $30 \%$ in den vier betrachteten Schularten (Berufliche Schulen, Real- und Volksschulen sowie Gymnasien) vertreten. Der Typus Vorgesetzter mit pädagogischer Verantwortung ist in Realschulen vergleichsweise selten anzutreffen. Nur 14\% der Personen dieser Gruppierung entfallen auf diese Schulart; jeweils zwischen $26 \%$ und 30\% sind in Volksschulen, Beruflichen Schulen und Gymnasien angesiedelt.

Bei der Analyse schulgrößenspezifischer Effekte bilden entsprechend der Scheffé-Prozedur die Pädagogische Führungskraft und der Vorgesetzte mit pädagogischer Verantwortung eine homogene Untergruppe, an deren Schulen rund 200 Schüler mehr unterrichtet werden ( $\mathrm{M}=858$ bzw. 875) als an denjenigen des Generalisten und der Lehrkraft mit Verwaltungsaufgaben, die eine weitere Gruppe bilden $(M=645$ bzw. 676). Der ClusterTypus Teamleiter ist an Schulen mit durchschnittlich 721 Schülern tätig.

\subsection{Subjektive Belastungs- und Zufriedenheitsurteile in Abhängigkeit der Clusterzugehörigkeit}

Erste Hinweise auf die vermuteten Zusammenhänge zwischen Ausprägungen des beruflichen Selbstverständnisses und der beruflichen Beanspruchung ergeben sich auf dimensionaler Ebene aus Tab. 2, die einige höchst signifikante Korrelationen zwischen den Rollensegmenten und den Facetten des Belastungs- und Zufriedenheitserlebens dokumentiert. Dabei gehen insbes. hohe Werte im Bereich Administration mit reduzierter Zufriedenheit und erhöhtem Belastungsempfinden einher.

Noch aufschlussreicher erweisen sich jedoch auf der typologischen Ebene die varianzanalytischen Vergleiche der Clustermittelwerte. Sie zeigen durchgängig statistisch signifikante wie auch praktisch bedeutsame Beanspruchungsdifferenzen mittlerer Effektstärke in Abhängigkeit der Berufsaufassung als der spezifischen Gesamtkonfiguration des subjektiven Rollenhaushalts. Wie Tab. 3 zu entnehmen ist, besitzen alle Cluster dasselbe Belastungsprofil, jedoch auf unterschiedlichem Niveau: Über alle Gruppen hinweg dominiert ein subjektiver Mangel an Anerkennung und spürbaren Erfolgserlebnissen im Beruf (Feedbackdefizit), gefolgt von Zeitstress und allgemeinen gesundheitlichen Befindlichkeitsstörungen sowie einem wahrgenommenen normativen Druck. Übereinstimmend mit den formulierten Erwartungen weisen aber diejenigen Gruppierungen, deren Berufsauffassung auf mangelnde Rollendistanz respektive auf ungelöste Rollenkonflikte schließen lässt, signifikant stärkere negative Beanspruchungsreaktionen auf als die anderen Leitungstypen: Die Scheffé-Prozedur klassifiziert die Cluster Lehrkraft mit Verwaltungsaufgaben und Generalist auf der einen Seite sowie die Cluster Pädagogische Führungskraft und Teamleiter auf der anderen Seite als homogene Untergruppen, die systematisch in allen belastungsbezogenen Erlebensqualitäten voneinander abweichen. Dabei bestehen die größten Unterschiede im Bereich normativen Drucks als derjenigen Belastungskategorie, die das Erleben von Überforderung angesichts unerfüllbarer externer Erwartungen und erdrückend hoher persönlicher Verantwortung erfasst. Der Cluster Vorgesetzter mit pädagogischer Verantwortung nimmt mit seinen Merkmalsausprägungen eine Mittelposition ein und wird in den einzelnen Belastungskategorien entweder als eigenständige Untergruppe klassifiziert oder der aus Lehrkraft und Generalist bestehenden Gruppe zugeordnet. 


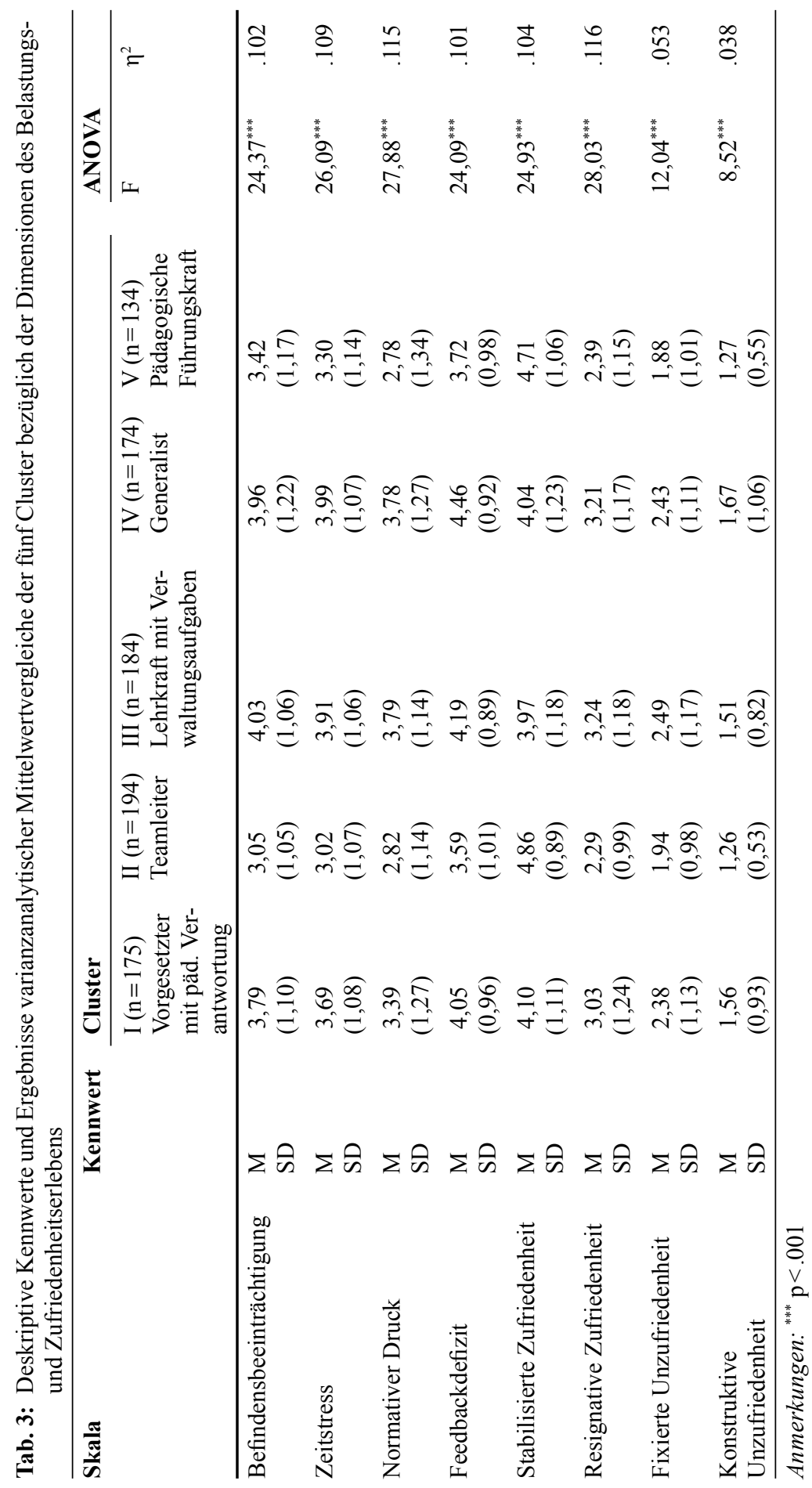


Hiermit konsistent fällen die Lehrkraft mit Verwaltungsaufgaben und der Generalist auch signifikant negativere Zufriedenheitsurteile als ihre Kollegen. ${ }^{4}$ Demnach stellt sich in diesen Clustern signifikant seltener eine stabilisierte Zufriedenheitsform ein, die in der Befriedigung eigener Bedürfnisse und Erwartungen wurzelt und auf Wahrung des Erreichten abzielt. Vielmehr berichten diese Personen in weit höherem Ausmaß von einer resignativen Absenkung des eigenen Anspruchsniveaus als Reaktion auf Soll-Ist-Diskrepanzen, die in der Vergangenheit nicht mithilfe geeigneter Problemlösungsmechanismen überwunden werden konnten. In den Bereichen der fixierten und konstruktiven Unzufriedenheit fallen die Unterschiede zu den übrigen Clustern zwar immer noch höchst signifikant aus, besitzen aber eine nur geringe Effektstärke. Folglich sehen sich insgesamt nur wenige Leitungskräfte in einer höchst unbefriedigenden und dabei ausweglosen Arbeitssituation gefangen oder wollen sich aus einer solchen Situation aktiv lösen, auch wenn die Cluster Pädagogische Führungskraft und Teamleiter noch seltener zu dieser Einschätzung gelangen als die übrigen Cluster.

\subsection{Kontrolle ausgewählter personen- und kontextbezogener Einflussfaktoren des} generellen Beanspruchungsniveaus

Um die Analyse clusterbedingter Gruppenunterschiede in der beruflichen Beanspruchung unter Ausschluss potenzieller konfundierender Variablen zu vertiefen, wurde der Einfluss der Variablen Schulart, Schulgröße, Dienstalter und Geschlecht auf die beiden beanspruchungsrelevanten Gesamtskalen mithilfe von Kovarianzanalysen kontrolliert.

Aus Tab. 4 geht zunächst hervor, dass die Mittelwerte des globalen Belastungserlebens clusterübergreifend um den theoretischen Mittelwert von 3,5 streuen. Dieses Ergebnis korrespondiert mit Studien, die eine im Vergleich zu Lehrkräften eher moderate Beanspruchung schulischer Leitungspersonen ermitteln (z.B. Kanders \& Rösner 2006). Zudem bewegt sich die globale Zufriedenheit auf erhöhtem bis hohem Niveau. Allerdings dokumentieren erneut die varianzanalytischen Vergleiche höchst signifikante Abweichungen in Abhängigkeit der Clusterzuordnung mit mittlerer und großer Effektstärke, welche die Typen Lehrkraft, Generalist und Vorgesetzter als bedeutsam unzufriedener ausweisen als die Typen Pädagogische Führungskraft und Teamleiter (homogene Untergruppen gemäß Tamhane-T2-Verfahren). Auch die subjektive Belastung erreicht bei diesen Gruppierungen deutlich höhere Werte, wobei der Typus Vorgesetzter hier eine eigenständige Position auf mittlerem Niveau einnimmt.

Erwartungsgemäß reduziert die Berücksichtigung der biografischen und kontextbezogenen Variablen die durch die Clusterzugehörigkeit aufgeklärten Varianzen in den Gesamtskalen. Dennoch bleiben jeweils substanzielle Gruppenunterschiede mit mittlerer Effektstärke erhalten. Wird das generelle Belastungserleben als Kriteriumsvariable betrachtet, lassen sich bedeutsame Effekte der Schulart $\left(F=21.83, p<.001, \eta^{2}=.026\right)$ und der Schulgröße $\left(\mathrm{F}=5.03, \mathrm{p}<.05, \eta^{2}=.006\right)$ nachweisen, während biografische Merkmale der Person ohne Auswirkungen bleiben. Fungiert dagegen die globale Zufriedenheit als Kriteriumsvariable, ist neben der Schulart $\left(\mathrm{F}=33.19, \mathrm{p}<.01, \eta^{2}=.039\right)$ und der Schulgröße $\left(\mathrm{F}=17,66, \mathrm{p}<.01, \eta^{2}=.021\right)$ auch das Geschlecht der Leitungspersonen statistisch relevant $\left(\mathrm{F}=6.20, \mathrm{p}<.05, \eta^{2}=.008\right)$. Demzufolge ist das Belastungserleben an Volksschulen sowie an kleinen Schulen besonders ausgeprägt. Die hier tätigen Leitungskräfte sind 


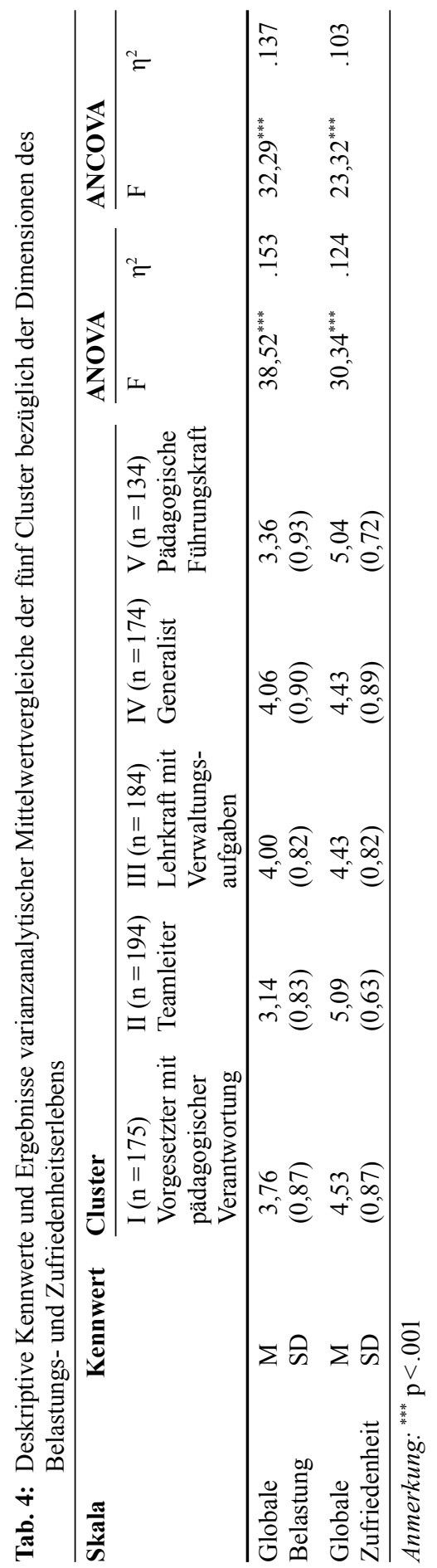


auch generell unzufriedener als die übrigen Mitglieder ihres Berufsstandes. Zudem fällt die Zufriedenheitsbilanz weiblicher Leitungskräfte in der Regel etwas günstiger aus als diejenige ihrer männlichen Kollegen.

\section{Diskussion und Ausblick}

Im Zentrum der hier berichteten Analysen stand die Frage, über welche Berufsauffassungen (im Sinne divergenter Interpretationsmuster des beruflichen Rollenhandelns) schulische Leitungspersonen verfügen und ob diese auch Unterschiede in den berufsbedingten Beanspruchungsprofilen begründen können. Mithilfe clusteranalytischer Verfahren ergab sich eine Binnendifferenzierung in fünf Gruppen von Leitungskräften, die systematisch in ihrer subjektiven Gewichtung der drei Rollensegmente Leadership, Kollegialität und Administration voneinander abweichen. Die so ermittelten Leitungstypen lassen sich sinnvoll in den gegenwärtigen Stand der Schulleitungsforschung integrieren, untermauern jedoch die Annahme, dass in dieser Berufsgruppe unterschiedlich akzentuierte berufliche Subkulturen koexistieren, die sich zum Teil kontextspezifisch ausbilden.

Resümierend lässt sich festhalten, dass mit Ausnahme des als Generalist bezeichneten Clusters, der in allen drei Rollensegmenten überdurchschnittlich hohe Ausprägungen besitzt, alle Leitungstypen mehr oder minder ausgeprägte Akzente bei der Wahrnehmung und Gestaltung ihres Amtes setzen. Dabei bildet sich auch ein Typus heraus, der dem in vorgängigen Studien skizzierten Bild eines im Rollenhandeln des Lehrerberufs verhafteten Primus Inter Pares mit umfänglichen Verwaltungsaufgaben entspricht (Typus Lehrkraft mit Verwaltungsaufgaben). Diese Gruppe distanziert sich von den für Leitungspositionen typischen Steuerungsfunktionen, räumt einem dem Autonomie-Paritätsmuster verpflichteten Auftreten einen hohen Stellenwert ein und gewichtet administrative Funktionen deutlich höher als Elemente der Mitarbeiterführung. Kontrastiert werden diese beiden Extremgruppen vom Typus der Pädagogischen Führungskraft, der seine strategische und für die schulische Entwicklung richtungsweisende Position stark hervorhebt, vom Typus des Teamleiters, der alle administrativen Elemente des Berufsbildes entschieden zurückweist und strategische Aufgaben partnerschaftlich-kollegial im Kollegenkreis zu bewältigen sucht, sowie vom Typus des Vorgesetzten mit pädagogischer Verantwortung, der auf Basis eines klaren hierarchischen Gefälles bemüht ist, aktiv-gestaltende Führungsaufgaben und reaktiv-vollziehende Verwaltungstätigkeiten auf moderatem Niveau auszutarieren.

Die Clusterzugehörigkeit erwies sich im Zuge $\chi^{2}$-basierter und varianzanalytischer Gruppenvergleiche entgegen der eingangs formulierten Annahmen nicht als von biografischen Personenmerkmalen abhängig, wohl aber erwartungskonform als beeinflusst von Art und Größe der geleiteten Schule. Dementsprechend bilden sich die Muster des Generalisten und der Lehrkraft mit Verwaltungsaufgaben vorrangig an Grund- und Hauptschulen aus, während sich die übrigen Cluster recht gleichmäßig auf Volks-, Real- und Berufliche Schulen sowie Gymnasien verteilen. Sie sind zudem eher an kleineren Schulen präsent, wohingegen die Typen pädagogische Führungskraft und Vorgesetzter mit pädagogischer Verantwortung an großen Schulen dominieren. 
Mit der Analyse des Zufriedenheits- und Belastungserlebens der Befragten wurden unter Verweis auf die Ergebnisse der Lehrerbelastungsforschung zudem zwei für die individuelle Gesundheit und Leistungsfähigkeit wichtige Zielgrößen des Leitungshandelns fokussiert. Es konnte gezeigt werden, dass unterschiedliche typologische Konfigurationen des beruflichen Selbstverständnisses über die betrachteten biografischen und kontextbezogenen Einflussfaktoren hinaus einen zusätzlichen Erklärungsbeitrag für die Ausprägungshöhe der subjektiven Belastung und Zufriedenheit im Beruf leisten.

Zwar deutet sich bei keinem der fünf Cluster eine Gefährdungslage an, die eine Einstufung der zugehörigen Schulleiter(innen) als gesundheitspsychologische Risikopopulation rechtfertigen würde (vgl. hierzu Schaarschmidt \& Fischer 2001), dennoch weisen die Cluster Generalist sowie Lehrkraft mit Verwaltungsaufgaben vergleichsweise ausgeprägte negative Beanspruchungsreaktionen auf. Beide Cluster nehmen nicht nur den stärksten zeitlichen Druck und die stärksten Beeinträchtigungen ihrer Gesundheit wahr, sondern fühlen sich auch in höherem Maße uneinlösbaren externen Forderungen ausgesetzt und verbuchen bei der täglichen Arbeit nur geringe Erfolgsrückmeldungen. Zudem berichten sie häufiger als andere Schulleiter(innen) von einer Minderung des eigenen beruflichen Anspruchsniveaus. Diese Befunde stützen die Annahme, dass zum einen der Versuch, konfligierenden Verhaltenserwartungen simultan und jeweils optimal zu entsprechen, sowie zum anderen die Unvereinbarkeit einer im Ausgangsberuf der Lehrkraft verhafteten Handlungsrationalität mit den Steuerungsaufgaben des Leitungsamtes in Resignation bzw. Selbstüberforderung münden können.

Dagegen gelingt es den Clustern pädagogische Führungskraft und Teamleiter offenbar, eigene Vorstellungen und fremddefinierte Handlungsanforderungen derart in ihrem beruflichen Selbstverständnis zu versöhnen, dass eine spannungs- und belastungsreduzierende Balance resultiert, die sich auch in überdurchschnittlichen Zufriedenheitswerten niederschlägt. Durch die günstigen beanspruchungsrelevanten Werte dieser Cluster wird die Bedeutung organisationspädagogischer Ansätze der Schulleitung unterstrichen (vgl. Rosenbusch 2005), die eine Konzentration des Leitungshandelns auf die pädagogische Arbeit der Schule einfordern und administrativen Tätigkeiten eine nachrangige, instrumentelle Funktion zuweisen.

Die signifikanten und praktisch bedeutsamen Effekte der Berufsauffassung auf die Beanspruchung bleiben auch erhalten, nachdem Einflüsse von Person und Kontext rechnerisch auspartialisiert wurden, um eine Konfundierung beider Konstrukte durch dritte Variablen zu kontrollieren. Erwartungsgemäß variiert die subjektive Belastungsintensität dabei in Abhängigkeit der Schulart und der Schulgröße; für die subjektive Zufriedenheitsbilanz ist darüber hinaus auch das Geschlecht der Leitungsperson relevant.

In Übereinstimmung mit transaktionalen Ansätzen der Belastungsforschung erweisen sich damit Personen- und Umweltvariablen als beanspruchungsrelevante Bedingungsfaktoren. Als Erkenntnisbeitrag der vorliegenden Studie mag gewertet werden, dass der individuellen Konfiguration des beruflichen Rollenhaushalts dabei eine gewichtige Bedeutung zukommt und fehlende Rollendistanz bzw. ungelöste Rollenkonflikte sich negativ auf das Belastungs- und Zufriedenheitsempfinden auswirken können. Einschränkend muss darauf verwiesen werden, dass in die Analysen bei Weitem nicht alle einflussträchtigen Merkmale des Handlungskontexts und der individuellen Handlungsvoraussetzungen einbezogen wurden. $\mathrm{Zu}$ denken ist hier beispielsweise an die Zusammensetzung und das 
Engagement des Lehrerkollegiums, das Einzugsgebiet der Schule oder die Arbeitsorganisation und Coping-Strategien der Leitungsperson. Diesen Einflussgrößen soll in weiterführenden Auswertungen im Rahmen des Forschungsprojekts nachgegangen werden. Darüber hinaus kann kritisch diskutiert werden, ob mit den drei zugrunde gelegten Rollensegmenten Leadership, Kollegialität und Administration das berufliche Rollenhandeln der Schulleitung bereits erschöpfend abgebildet wurde. Im operationalen Zugriff dieser Studie erschien es jedoch wichtiger, wenige trennscharfe als viele stark ausdifferenzierte, aber nicht überschneidungsfreie Konstrukte zu erfassen.

Um das Instrument einer weiteren Validierung zu unterziehen, bedarf es deshalb einer Replikation der Befunde. Wünschenswert erscheint diese auch in Anbetracht der eingeschränkten Repräsentativität der Stichprobe, die nicht nach dem Zufallsprinzip, sondern im Quotenverfahren gezogen wurde, um Schulartvergleiche zu ermöglichen.

Schließlich ist zu bedenken, dass die im Rahmen einer Befragung erhobenen Selbsteinschätzungen der Leitungspersonen keine unmittelbare Handlungsvalidierung ermöglichen. Die Frage, ob sich unterschiedlich akzentuierte Berufsaufassungen auch in unterschiedlichen Mustern des faktischen Leitungshandelns niederschlagen, kann derzeit nur mit Verweis auf die in anderen Studien nachgewiesene Handlungswirksamkeit berufsbezogener Sichtweisen beantwortet werden (vgl. zusammenfassend Seifried 2008). Dringend erforderlich erscheinen deshalb Studien, welche die tatsächlich realisierten Formen des Leitungshandelns beobachten oder aus Sicht der Mitarbeiter beurteilen und die weiterhin den Erfolg des Leitungshandelns nicht nur an subjektiven Urteilen zur persönlichen Arbeitsqualität, sondern auch an Zielmerkmalen auf den Wirkungsebenen der Lehrkräfte (beispielsweise wahrgenommene Unterstützung, Teamstrukturen) und des Unterrichts (Lernprozesse und -ergebnisse) messen. Zu diesem Zweck ist bereits eine Anschlusserhebung bei den Lehrkräften und Schüler(inne)n der untersuchten Schulen angelaufen.

\section{Anmerkungen}

1 Das Modell berücksichtigt zudem Rückkoppelungen zwischen der Leitungstätigkeit und ihren Bestimmungsgrößen. Dabei sind die Rückwirkungen individuellen Schulleitungshandelns auf die gesellschaftlichen Rahmenbedingungen als vergleichsweise gering einzustufen (was in der Abbildung durch einen dünnen Pfeil indiziert wird). Sie erfolgen beispielsweise in Form der Eltern- und Öffentlichkeitsarbeit einer Leitungsperson.

2 Das Ausprägungsniveau des Vorgesetzten-Clusters auf der Skala Administration rückt diesen in die Nähe der Lehrkraft mit Verwaltungsaufgaben. Beide Cluster bilden auf dieser Skala laut Scheffé-Test eine homogene Untergruppe.

3 Entsprechend ihrer Mittelwerte bilden diese beiden Cluster laut Scheffé-Test die einzige homogene Untergruppe in der Dimension Kollegialität.

4 Wiederum bilden die Pädagogische Führungskraft und der Teamleiter eine erste, der Generalisten und die Lehrkraft mit Verwaltungsaufgaben eine zweite homogene Untergruppe. Die Zuordnung des Vorgesetzten-Typus in diese beiden Gruppen variiert von Subskala zu Subskala. 


\section{Literatur}

Altrichter, H., \& Eder, F. (2004). Das ,Autonomie-Paritäts-Muster‘ als Innovationsbarriere? In H. G. Holtappels (Hrsg.), Schulprogramme - Instrumente der Schulentwicklung (S. 195-221). Weinheim: Juventa.

Altrichter, H., \& Rauscher, E. (2008). Schulleitung und neue Steuerungskultur. In J. Warwas \& D. Sembill (Hrsg.), Zeitgemäße Führung - zeitgemäßer Unterricht (S. 29-44). Hohengehren: Schneider Verlag.

Ammann, T. (2004). Zur Berufszufriedenheit von Lehrerinnen. Erfahrungsbilanzen in der mittleren Berufsphase. Bad Heilbrunn/Obb.: Klinkhardt.

Bacher, J. (1996). Clusteranalyse. Anwendungsorientierte Einführung. München: Oldenbourg.

Baethge, M., Achtenhagen, F., Arends, L., Babic, E., Baethge-Kinsky, V., \& Weber, S. (2006). Berufsbildungs-PISA. Machbarkeitsstudie. Stuttgart: Franz Steiner.

Baumert, J., \& Leschinsky, A. (1986). Berufliches Selbstverständnis und Einflussmöglichkeiten von Schulleitern. Ergebnisse einer Schulleiterbefragung. Zeitschrift für Pädagogik, 32(2), 247-266.

Bieri, T. (2006). Lehrpersonen - hoch belastet und trotzdem zufrieden? Bern: Haupt.

Bonsen, M. (2006). Wirksame Schulleitung - Forschungsergebnisse. In H. Buchen \& H.-G. Rolff (Hrsg.), Professionswissen Schulleitung (S. 193-228). Weinheim: Beltz.

Bortz, J. (2005). Statistik für Human- und Sozialwissenschaftler (6. vollst. überarb. und aktualisierte Aufl.). Heidelberg: Springer.

Brägger, G., \& Posse, N. (2007). Instrumente für die Qualitätsentwicklung und Evaluation in Schulen (IQES). Wie Schulen durch eine integrierte Gesundheits- und Qualitätsförderung besser werden können. Bern: hep-Verlag.

Bruggemann, A., Groskurth, P., \& Ulich, E. (1975). Arbeitszufriedenheit. Bern: Huber.

Buchen, H., \& Rolff, H.-G. (Hrsg.) (2006). Professionswissen Schulleitung. Weinheim: Beltz.

Dann, H., Humpert, W., Krause, F., Kügelgen, T. von, Rimele, W., \& Tennstädt, K. (2007). Subjektive Aspekte des Lehrerberufs. In A. Glöckner-Rist (Hrsg.), ZUMA-Informationssystem. Elektronisches Handbuch sozialwissenschaftlicher Erhebungsinstrumente. ZIS Version 10.00. Mannheim: ZUMA.

Dubs, R. (2005). Die Führung einer Schule. Leadership und Management (2., überarb. Aufl.). Zürich: SKV.

Esslinger, I. (2002). Berufsverständnis und Schulentwicklung: Ein Passungsverhältnis? Eine empirische Untersuchung zu schulentwicklungsrelevanten Berufsauffassungen von Lehrerinnen und Lehrern. Bad Heilbrunn/Obb.: Klinkhardt.

Fend, H. (2006). Neue Theorie der Schule. Einführung in das Verstehen von Bildungssystemen. Wiesbaden: VS Verlag für Sozialwissenschaften.

Forberg, A. (1997). Rollen- und Führungsverständnis von Schulleiterinnen beruflicher Schulen. Eine berufsbiographisch-orientierte Untersuchung. Weinheim: Dt. Studien Verlag.

Füssel, H.-P. (1998). Schulleitung zwischen staatlicher Steuerung und schulischer Handlungsautonomie. In H. Ackermann \& J. Wissinger (Hrsg.), Schulqualität managen - von der Verwaltung der Schule zur Entwicklung von Schulqualität (S. 149-159). Neuwied: Luchterhand.

Hobeck, D. (2005). Die Unterrepräsentanz von Frauen in Schulleitungen - Mögliche Ursachen aus naturwissenschaftlich-anthropologischer Perspektive. Frankfurt a. M.: Peter Lang.

Kanders, M., \& Rösner, E. (2006). Das Bild der Schule im Spiegel der Lehrermeinung - Ergebnisse der 3. IFS-Lehrerbefragung 2006. In H. G. Holtappels, W. Bos, H. Pfeiffer, H.-G. Rolff \& R. Schulz-Zander (Hrsg.), Jahrbuch der Schulentwicklung: Daten, Beispiele und Perspektiven. $B d .14$ (S. 11-48). Weinheim: Juventa.

Kluge, S. (2000). Empirisch begründete Typenbildung in der qualitativen Sozialforschung. In Forum Qualitative Sozialforschung, Theorien, Methoden, Anwendungen. http://217.160.35.246/fqstexte/1-00/1-00kluge-d.htm. Zugriff am 30. April 2009. 
Körner, S. (2003). Das Phänomen Burnout am Arbeitsplatz Schule - Ein empirischer Beitrag zur Beschreibung des Burnout-Syndroms und seiner Verbreitung sowie zur Analyse von Zusammenhängen und potentiellen Einflussfaktoren auf das Ausbrennen von Gymnasiallehrern. Berlin: Logos.

Kranz, T. (2007). Das Führungsverständnis angehender Schulleiterinnen und Schulleiter. Norderstedt: Books on Demand. ((GmbH streichen))

Krappmann, L. (2005). Soziologische Dimensionen der Identität. Strukturelle Bedingungen für die Teilnahme an Interaktionsprozessen (10. Aufl.). Stuttgart: Klett-Cotta.

Languth, M. (2006). Schulleiterinnen und Schulleiter im Spannungsverhältnis zwischen programmatischen Zielvorgaben und alltäglicher Praxis. Eine empirische Untersuchung zur Berufsauffassung von Schulleiterinnen und Schulleitern. Dissertation an der Georg-August-Universität Göttingen. http://webdoc.sub.gwdg.de/diss/2007/languth/languth.pdf. Zugriff am 30. April 2009.

Lazarus, R. S. (1990). Streß und Streßbewältigung - Ein Paradigma. In S. H. Filipp (Hrsg.), Kritische Lebensereignisse (S. 198-232). München: Beltz PVU.

Mayer, A. K., Filipp, S. H., \& Aymanns, P. (2004). Formen der Lebensbewältigung älterer Menschen aus Sicht ihrer erwachsenen Kinder und die Qualität der Eltern-Kind-Beziehung: Eine clusteranalytische Untersuchung. Zeitschrift für Entwicklungspsychologie und Pädagogische Psychologie, 36(2), 95-104.

Neuberger, O. (2002). Führen und führen lassen. Ansätze, Ergebnisse und Kritik der Führungsforschung. (6., völlig neu bearb. und erw. Aufl.). Stuttgart: Lucius \& Lucius.

Roggenbuck-Jagau, I. (2005). Berufsverständnis und Professionalisierung von Schulleitern. Wiesbaden: VS Verlag für Sozialwissenschaften.

Rolff, H.-G. (2006). Schulentwicklung, Schulprogramm und Steuergruppe. In H. Buchen \& H.-G. Rolff (Hrsg.), Professionswissen Schulleitung (S. 296-364). Weinheim: Beltz.

Rosenbusch, H. S. (2005). Organisationspädagogik der Schule. Grundlagen pädagogischen Führungshandelns. Neuwied: Luchterhand.

Rosenbusch, H. S., Braun-Bau, S., \& Warwas, J. (2006). Schulleitungstätigkeit an bayerischen Grund-, Haupt- und Realschulen. Bestandsaufnahme und Vorschläge für eine Neuorientierung (Kurzfassung). Die Schulleitung, 33(4), 4-11.

Rudow, B. (2000). Der Arbeits- und Gesundheitsschutz im Lehrerberuf. Gefährdungsbeurteilung der Arbeit von Lehrerinnen und Lehrern. Ludwigsburg: Süddeutscher Pädagogischer Verlag.

Schaarschmidt, U., \& Fischer, A. (2001). Bewältigungsmuster im Beruf. Persönlichkeitsunterschiede in der Auseinandersetzung mit der Arbeitsbelastung. Göttingen: Vandenhoeck \& Ruprecht.

Schmieta, M. (2001). Die Relevanz von Persönlichkeitsmerkmalen und beruflichen Einstellungen bei der Entwicklung von Burnout. Ein Vergleich zwischen Beratungslehrern und Lehrern ohne Zusatzausbildung (Studien zur Stressforschung, Bd. 11). Hamburg: Dr. Kovač.

Schwarzer, R., \& Jerusalem, M. (2001). Skalen zur Erfassung von Lehrer- und Schülermerkmalen. Dokumentation der psychometrischen Verfahren im Rahmen der Wissenschaftlichen Begleitung des Modellversuchs Selbstwirksame Schulen. Korrigierte Web-Version. http://web.fuberlin.de/gesund/schulen/skalen.htm. Zugriff am 30. April 2009.

Seifried, J. (2008). Unterricht aus der Sicht von Handelslehrern. Frankfurt a. M.: Peter Lang.

Seitz, H., \& Capaul, R. (2005). Schulführung und Schulentwicklung. Theoretische Grundlagen und Empfehlungen für die Praxis. Bern: Haupt.

Sembill, D. (2006). Zeitlebens Lebenszeit. In G. Minnameier \& E. Wuttke (Hrsg.), Berufs- und wirtschaftspädagogische Grundlagenforschung - Lehr-Lern-Prozesse und Kompetenzdiagnostik (S. 177-194). Frankfurt a. M.: Peter Lang.

Steinmann, H., \& Schreyögg, G. (2005). Management. Grundlagen der Unternehmensführung. Konzepte - Funktionen - Fallstudien (6., vollst. überarb. Aufl.). Wiesbaden: Gabler. 
Storath, R. (1995). „Praxisschock“ bei Schulleitern? Eine Untersuchung zur Rollenfindung neu ernannter Schulleiter. Neuwied: Luchterhand.

Terhart, E. (1996). Berufskultur und professionelles Handeln bei Lehrern. In A. Combe \& W. Helsper (Hrsg.), Pädagogische Professionalität. Untersuchungen zum Typus pädagogischen Handelns (2. Aufl.) (S. 448-471). Frankfurt a. M.: Suhrkamp.

Terhart, E. (1997). Schulleitungshandeln zwischen Organisation und Erziehung. In J. Wissinger (Hrsg.), Schulleitung als pädagogisches Handeln - Schulleiter-Handbuch, Bd. 83 (S. 7-20). München: Oldenbourg.

Warwas, J. (in Vorb.). Berufliches Selbstverständnis und berufsbedingte Beanspruchungsmuster pädagogischer Führungskräfte. Bisher unveröffentl. Dissertation Bamberg: Otto-Friedrich-Universität.

Werle, P. (2001). Zum beruflichen Selbstkonzept des Schulleiters. Eine Untersuchung zum beruflichen Selbstbild und Selbstverständnis von Schulleiterinnen und Schulleitern sowie deren Bedürfnisse und Erwartungen an die Schulleiterfortbildung. Saarbrücken: Conte.

Wissinger, J. (1996). Perspektiven schulischen Führungshandelns. Eine Untersuchung über das Selbstverständnis von SchulleiterInnen. Weinheim: Juventa.

Zlatkin-Troitschanskaia, O. (2006). Steuerbarkeit von Bildungssystemen mittels politischer Reformstrategien - Interdisziplinäre theoretische Analyse und empirische Studie zur Erweiterung der Autonomie im öffentlichen Schulwesen. Frankfurt a. M.: Peter Lang. 